Z Herz- Thorax- Gefäßchir 2017 · 31:77 DOI 10.1007/s00398-017-0150-5

Online publiziert: 7. März 2017

๑) Springer Medizin Verlag GmbH 2017

CrossMark

K. Kallenbach

Haerzzenter - INCCI, Luxembourg, Luxemburg

\title{
Operationsplanung per iPhone: die TEVAR-App
}

dene Produkte dargestellt. Diese Produkte werden dezidiert beschrieben und deren Anwendung erklärt („Instruction to use“), die radiologischen Marker dargestellt, Fotos und z. T. kurze Filme über die Implantation angeboten. Bei einigen Grafts ist sogar ein „Troubleshooting“ verfügbar, falls es bei der Handhabung Probleme gibt! Und mithilfe des „calculator" lassen sich, nach Eingabe individueller Aortenpathologien und Gefäßdiameter, wie sie im CT vermessen wurden, verschiedene Behandlungsoptionen simulieren, sodass für eine eingegebene Aortenpathologie bis zu 5 verschiedene Produkte zur Auswahl stehen - mit proximalem und distalem Diameter, „oversizing“, Länge und Angabe des äußeren Diameters. Auch die Kombination verschiedener Stents bei unterschiedlichen Landungszonen wird in die Planung einbezogen.

Die Anwendung ist einfach und selbsterklärend, sodass ich umgehend für den nächsten Patienten diese App zur Behandlungsplanung genutzt habe - so, wie vom Erfinder vorgeschlagen!

Fazit: Höchste Zeit, sich die App runterzuladen, falls noch nicht geschehen; oder sind Sie etwa nicht mehr zeitgemäß? Natürlich können Sie auch zunächst den Artikel in dieser aktuellen Ausgabe der Zeitschrift für Herz-, Thorax-und Gefäßchirurgie lesen, sozusagen als Motivationshilfe ...

Mit herzlichen Grüßen Ihr

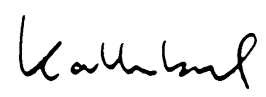

Prof. Dr. Klaus Kallenbach

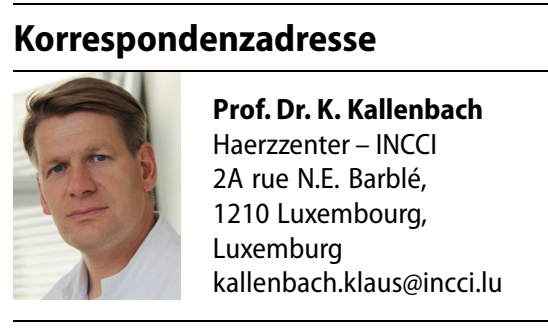

Interessenkonflikt. K. Kallenbach gibt an, dass kein Interessenkonflikt besteht. 